\title{
Orthopäden brauchen am meisten Kaffee
}

\author{
Von den vielen unbeantworteten Fragen, die in Krankenhäusern \\ immer noch offen sind, ist eine jetzt endgültig beantwortet: Am \\ meisten Kaffee trinken die orthopädischen Chirurgen.
}

_ Seitdem das erste Kaffeehaus in Konstantinopel 1555 etabliert und die Türken 1683 Wien belagerten, eroberte das schwarze Getränk auch Europa im Sturm. Ohne die stimulierende Wirkung dieses (Über-)Lebenselixiers ist die Arbeitswelt heute kaum noch vorstellbar. Der Konsum könnte somit ein Indikator für die Stressbelastung einzelner Fachgruppen im Krankenhaus sein.

Über das elektronische Bezahlsystem wurde im Lauf des Jahres 2014 der tägliche Kaffeekonsum der 766 Ärzte eines großen akademischen Lehrkrankenhauses in der Schweiz erfasst. Die Daten umfassten Berufsgruppe und Spezialisierung, Abteilungszugehörigkeit, hierarchische Position, Alter und Geschlecht. Ausgewertet wurden nur die anonymisierten Daten für die Ärzte. Darunter waren 201 Internisten, 76 Allgemeinchirurgen, 67 Anästhesisten, 54 Radiologen, 48 Orthopäden, 43 Gynäkologen, 36 Neurologen, 23 Neurochirur- gen und 96 Ärzte anderer Spezialisierung. 84\% der Ärzte ( $=644)$ kauften Kaffee in einer der Kantinen des Krankenhauses. Im gesamten Jahr 2014 wurden 70.772 Tassen konsumiert.

In einer univariaten Varianzanalyse ergab sich eine signifikante Assoziation zwischen Spezialisierung und dem jährlichen Kaffeekonsum. An der Spitze standen die orthopädischen Chirurgen mit einem Konsum von 189 Tassen pro Person, gefolgt von Radiologen mit 177 und Allgemeinchirurgen mit 167 Tassen. Erstaunlicherweise belegten die Anästhesisten mit 39 Tassen die letzte Position - zumindest in der Kantine. Männer tranken im Schnitt 128 Tassen, deutlich mehr als Frauen mit 86. Noch ausgeprägter war dieser Unterschied bei den Espressos (27 vs. 10). Analysierte man die hierarchische Position, so lagen die Chef- und Fachärzte mit 140 Tassen deutlich vor den jungen Assistenten mit nur 95 Tassen.

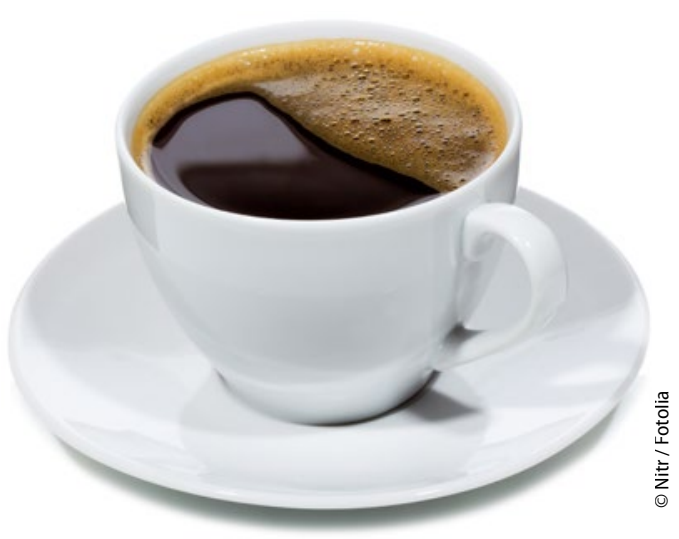

Geliebter, flüssiger, schwarzer Stimulus.

- Giesinger Ket al. Black medicine: an observational study of doctors' coffee purchasing patterns at work. BMJ. 2015;351:h6446

\section{KOMMENTAR}

Ob die Ärzte aus der oberen Hierarchie mehr Stimulation brauchen als die Jungen oder einfach mehr Zeit für Kaffeepausen haben, bleibt bei der Untersuchung offen. Immerhin lagen sie beim Spendieren von Kaffeerunden meilenweit vor den Jungärzten. Das wäre aber auch noch schöner gewesen.

Prof. Dr. med. H. S. FüeßI

\section{Die Ablehnung der Ablehnung wissenschaftlicher Artikel}

Wissenschaftliche Autoren wollen ihre Publikationen in Journalen mit möglichst hohem Impact Factor publizieren. Diese lehnen aber leider $80 \%$ der eingereichten Manuskripte ab. Um diese narzisstische Kränkung zu vermeiden, schlagen zwei Psychiater aus Sydney eine einfache „Ablehnung der Ablehnung" vor. Sie liefern sogar einen Formbrief!

Vielen Dank für die Ablehnung unseres Manuskripts in der Anlage. Leider können wir Ihre Ablehnung derzeit nicht akzeptieren. Wie Sie sicher wissen, erhalten wir jedes Jahr zahlreiche Ablehnungen und sind schlichtweg nicht in der Lage, alle zu akzeptieren. Wegen des wachsenden Drucks bezüglich hoher Zitationszahlen und einer starken Konkurrenz um Fördermittel können wir in der Regel nur etwa 30\% der eingehenden Ablehnungen annehmen. Bitte betrachten Sie diese Entscheidung nicht als Bewertung Ihrer Arbeit. Das Niveau einiger eingegangenen Ablehnungen ist sehr hoch.

Als Begründung für diese Ablehnung dürfen wir anführen, dass Gutachter 1 offensichtlich die Genialität der Studie nicht verstanden hat. Seine Aussage, wonach die Studie weder neu noch interessant sei und nichts zur Erweiterung des Wissens auf diesem Gebiet beitrage, reicht nicht hin. Auch die lateinischen Zitate von Gutachter 2 trugen zur Nichtakzeptanz Ihrer Ablehnung bei.

Wir wünschen Ihnen und Ihrem Gutachterteam viel Erfolg bei zukünftigen Ablehnungen und hoffen, dass Sie diese anderswo unterbringen können. Wir erlauben uns, in der Anlage Namen und Adressen einiger Autoren zu übersenden, die bereits mehrfach Ablehnungen von einflussreichen Journalen akzeptiert haben.

Bitte betrachten Sie unsere Entscheidung als endgültig. Wir haben die finale Fassung des Manuskripts im Original zusammen mit dem unterschriebenen Copyright-Formular hochgeladen und hoffen auf baldige Zusendung der Druckfahnen.

Ein brillianter Vorschlag direkt aus der australischen Psychiatrie!

- Chapman C, Slade T. Rejection of rejection: a novel approach to overcoming barriers to publication. BMJ 2015;351:h6326

Prof. Dr. med. H. S. FüeßI 